\title{
CSEM Forward and Adjoint Modeling Using Correspondence Principle
}

\author{
Adriany Tiffany Moura Reis* (UFPA, Faculdade de Geofísica, Belém (PA)), Jessé Carvalho Costa (UFPA, Faculdade de
} Geofísica, Belém (PA), Brasil \& INCT-GP)

Copyright 2019, SBGf - Sociedade Brasileira de Geofísica

This paper was prepared for presentation during the $16^{\text {th }}$ International Congress of the Geophysical Society held in Rio de Janeiro, Brazil, 19 to 22 August 2019.

Contents of this paper were reviewed by the Technical Committee of the $16^{\text {th }}$ International Congress of the Brazilian Geophysical Societyldeas and concepts of the text are authors' responsibility and do not necessarily represent any position of the SBGf, its officers or members. Electronic reproduction or storage of any part of this paper for commercial purposes without the written consent of the Brazilian Geophysical Society is prohibited.

\section{Abstract}

Modeling and inversion of electromagnetic (EM) data is, traditionally, implemented in frequency domain. For modeling of each single frequency response, it is necessary solution of a large linear system. For the inversion of CSEM data, the adjoint state method is applied to compute the gradient of an objective function, which essentially requires an additional frequency domain modeling. We discuss the implementation of forward and adjoint modeling of EM data in time domain by applying the correspondence principle. This approach transforms the Maxwell equations from a quasi-static regime, which describes EM fields in an electrically conductive medium, to corresponding Maxwell equations in a fictitious domain, which describes EM fields in a nonconductive medium. Forward and adjoint modeling in the fictitious domain are straightforward and efficient. In a single run because the EM fields can be computed for the whole large frequency band. Our 3D implementation was validated for using 2.5D and 3D models. We present the results of forward and adjoint modeling for a 3D resistivity model, based on the synthetic model Marlim R3D. Our numerical experiments show that the gradient of the leastsquares objective function for this synthetic data presents sufficient sensitivity to discriminate the reservoir region.

\section{Introduction}

CSEM is a low frequency exploration method, displacement currents are negligible, and the electromagnetic field behaviour follows a diffusive regime in time domain (Mittet, 2010). An alternative approach for time domain modeling of EM fields was proposed by Lee et al. (1989), where the electromagnetic diffusive equations are transformed to a wave equation regime. The solution of EM fields in the wave domain are later converted into time domain diffuse EM fields. In a later development, de Hoop (1996) proved the Correspondence Principle, which establishes a unique relationship between electromagnetic wavefields, in a fictitious dielectric media, to the diffusive electromagnetic fields, in conductive media. Mittet (2010) used the correspondence principle to performe EM modeling of CSEM data by finite difference in time domain.

The modeling of multi-frequency EM data using the correspondence principle requires a carefull treatment of the source pulse in the fictitious domain in orde to honor field data. Mittet (2010) uses a semi-analytical approach expanding the source pulse using the derivative of a Gaussian pulse. We propose an alternative approach to extract the pulse in the fictitious domain from frequency domain data using preconditioned conjugate gradient method. Using Ricker pulse as a preconditioner which reduces the pulse width and warrants a causal response.

In order to improve accuracy of EM modeling using the correspondence principle the treatment of the boundary conditions is an important issue. At the top, quasistatic boundary conditions are implement in the Fourier domain (Wang and Hohmann, 1993; Mittet, 2010). The other boundaries require efficient absorbing boundary conditions. We implemented Convolutional Perfect Matched Layer (CPML) boundary conditions (Roden et al., 2000; Komatitsch and Martin, 2007; de la Kethulle de Ryhove and Mittet, 2014).

We discuss the derivation and implementation of forward and adjoint modeling in the fictious domain and their application to compute the gradient of least-squares functionals for EM inversion applications. We present numerical experiments which show the effectiveness of the algorithm for modeling environments with complex geometry and anisotropic conductivity property. Finally, we present the computation of the gradient for the EM inversion on a realistic 3D synthetic model.

\section{Forward modeling using the Correspondence Principle}

We start from the diffusive Maxwell equations in the time domain in indicial notation, (Maaø, 2007):

$$
\begin{aligned}
-\varepsilon_{i j k} \frac{\partial H_{k}(x, t)}{\partial x_{j}}+\sigma_{i j}(x) E_{j}(x, t) & =-J_{i}(x, t), \\
\varepsilon_{i j k} \frac{\partial E_{k}(x, t)}{\partial x_{j}}+\mu \frac{\partial H_{i}(x, t)}{\partial t} & =-K_{i}(x, t),
\end{aligned}
$$

where $E_{k}(x, t)$ and $H_{k}(x, t)$ represent the components of electric and magnetic fields, respectively, $\sigma_{i j}(x)$ is anisotropic conductivity tensor and $\mu$ is the magnetic permeability; the sources are represented by $J_{i}(x, t)$ and $K_{i}(x, t)$, the electric and magnetic current density vectors, respectively, and $\varepsilon_{i j k}$ is permutation symbol.

Applying the forward Fourier transform,

$$
F(\omega)=\int_{-\infty}^{+\infty} d t f(t) e^{i \omega t}
$$

we obtain the Maxwell equations in the frequency domain, 


$$
\begin{aligned}
-\varepsilon_{i j k} \frac{\partial H_{k}(x, \omega)}{\partial x_{j}}+\sigma_{i j}(x) E_{j}(x, \omega) & =-J_{i}(x, \omega), \\
\varepsilon_{i j k} \frac{\partial E_{k}(x, \omega)}{\partial x_{j}}-i \omega \mu H_{i}(x, \omega) & =-K_{i}(x, \omega) .
\end{aligned}
$$

The correspondence principle establishes the relationship between equations (4) and (5) with the Maxwell equations in a dielectric medium:

$$
\begin{aligned}
-\varepsilon_{i j k} \frac{\partial H_{k}(x, \omega)}{\partial x_{j}}-i \omega \varepsilon_{i j}^{\prime}(x) E_{j}(x, \omega) & =-J_{i}(x, \omega), \\
\varepsilon_{i j k} \frac{\partial E_{k}(x, \omega)}{\partial x_{j}}-i \omega \mu H_{i}(x, \omega) & =-K_{i}(x, \omega) .
\end{aligned}
$$

Following (de Hoop, 1996), defining the fictitious dielectric tensor $\varepsilon_{i j}^{\prime}(x)$ by

and a complex frequency $\omega^{\prime}$,

$$
\sigma_{i j}(x)=2 \omega_{0} \varepsilon_{i j}^{\prime}(x),
$$

$$
\omega^{\prime}=(i+1) \sqrt{\omega \omega_{0}},
$$

the correspondence principle follows from the substitution of equations (8) and (9) into (4) and (5). Finally, after the following identifications for the fictitious EM fields and sources:

$$
\begin{aligned}
E_{k}^{\prime}\left(x, \omega^{\prime}\right) & =E_{k}(x, \omega), \\
H_{k}^{\prime}\left(x, \omega^{\prime}\right) & =\sqrt{\frac{-i \omega}{2 \omega_{0}}} H_{k}(x, \omega), \\
J_{k}^{\prime}\left(x, \omega^{\prime}\right) & =\sqrt{\frac{-i \omega}{2 \omega_{0}}} J_{k}(x, \omega), \\
K_{k}^{\prime}\left(x, \omega^{\prime}\right) & =K_{k}(x, \omega),
\end{aligned}
$$

one obtains (6) and (7) in the fictitious domain.

In order to obtain the corresponding the EM equations in fictitious time domain, we apply the inverse Fourier transform:

$$
\begin{gathered}
f\left(t^{\prime}\right)=\frac{1}{2 \pi} \int_{-\infty}^{+\infty} d \omega^{\prime} F\left(\omega^{\prime}\right) e^{-i \omega^{\prime} t^{\prime}}, \\
-\varepsilon_{i j k} \frac{\partial H_{k}^{\prime}\left(x, t^{\prime}\right)}{\partial x_{j}}+\varepsilon_{i j}^{\prime}(x) \frac{\partial E_{j}^{\prime}\left(x, t^{\prime}\right)}{\partial t^{\prime}}=-J_{i}^{\prime}\left(x, t^{\prime}\right), \\
\varepsilon_{i j k} \frac{\partial E_{k}^{\prime}\left(x, t^{\prime}\right)}{\partial x_{j}}+\mu \frac{\partial H_{i}^{\prime}\left(x, t^{\prime}\right)}{\partial t^{\prime}}=-K_{i}^{\prime}\left(x, t^{\prime}\right) .
\end{gathered}
$$

These are the equations to be solved by the Finite Difference Time Domain (FDTD). Certainly these equations do not represent observable fields, however, they have all the necessary information to retrieve the fields in the diffusive domain.

In order to obtain the Green's function associated to real conductive medium in frequency domain it is necessary to convert the fields and sources back from the fictitious time domain to the diffusive regime using the following relations (Mittet, 2010):

$$
\begin{array}{r}
J_{n}^{T}(\omega)=\sqrt{\frac{-2 \omega_{0}}{i \omega}} \int_{0}^{T} d t^{\prime} J_{n}^{\prime}\left(t^{\prime}\right) e^{-\sqrt{\omega \omega_{0}} t^{\prime}} e^{i \sqrt{\omega \omega_{0} t^{\prime}}} \\
E_{k}(x, \omega)=\int_{0}^{T} d t^{\prime} E_{k}^{\prime}\left(x, t^{\prime}\right) e^{-\sqrt{\omega \omega_{0} t^{\prime}}} e^{i \sqrt{\omega \omega_{0}} t^{\prime}}
\end{array}
$$

to finally obtain the Green's function

$$
G_{k n}^{E J}\left(x, \omega \mid x_{s}\right)=\frac{E_{k}(x, \omega)}{J_{n}^{T}(\omega)} .
$$

\section{CSEM numerical modeling}

The finite difference modeling of Maxwell equations in fictitious uses a staggered grid (Yee, 1966; Schild, 2009) with second order in time and fourth order in space. We used the optimized coefficients proposed by Holberg (1987); Mittet (2010) to reduce numerical dispersion, assuring a relative error between the phase and group velocities less than 0,003 .

The boundary condition between air and water was implemented using quasi-static approximation derived by Wang and Hohmann (1993) and discussed in Mittet (2010). For our high-order FD scheme we need to evaluate the following recursive equations:

$$
\begin{gathered}
\tilde{H}_{2}\left(x_{1}=-\left(i+\frac{1}{2}\right) \Delta x_{1}, \kappa_{2}, \kappa_{3}\right)= \\
e^{-\sqrt{\kappa_{2}^{2}+\kappa_{3}^{2}} i \Delta x_{1}} \tilde{H}_{2}\left(x_{1}=-\frac{\Delta x_{1}}{2}, \kappa_{2}, \kappa_{3}\right) \\
\tilde{H}_{3}\left(x_{1}=-\left(i+\frac{1}{2}\right) \Delta x_{1}, \kappa_{2}, \kappa_{3}\right)= \\
e^{-\sqrt{\kappa_{2}^{2}+\kappa_{3}^{2}} i \Delta x_{1}} \tilde{H}_{3}\left(x_{1}=-\frac{\Delta x_{1}}{2} \kappa_{2}, \kappa_{3}\right) \\
\tilde{E}_{2}\left(x_{1}=-i \Delta x_{1}, \kappa_{2}, \kappa_{3}\right)=e^{-\sqrt{\kappa_{2}^{2}+\kappa_{3}^{2}} i \Delta x_{1}} \tilde{E}_{2}\left(x_{1}=0, \kappa_{2}, \kappa_{3}\right) \\
\tilde{E}_{3}\left(x_{1}=-i \Delta x_{1}, \kappa_{2}, \kappa_{3}\right)=e^{-\sqrt{\kappa_{2}^{2}+\kappa_{3}^{2}} i \Delta x_{1}} \tilde{E}_{3}\left(x_{1}=0, \kappa_{2}, \kappa_{3}\right) .
\end{gathered}
$$

To assure convergence and accuracy, we enforced the stability condition (Mittet, 2010) and a space sampling interval smaller than a quarter of the minimum wavelength.

To avoid spurious reflections from all the other boundaries, we used the Convolutional Perfectly Matched Layer (CPML) method. In order implement the CPML we extended the model with boundary layers of eleven grid points. We adapted the scheme proposed by Komatitsch and Martin (2007) to the electromagnetic case. The attenuation variable, $s_{i}(t)$ is defined by

$$
s_{i}(\omega)=k_{i}+\frac{d_{i}}{\alpha_{i}+i \omega},
$$

where $k_{i}$ and $\alpha_{i}$ are equal to 1 and 0 . The attenuation follows a parabolic profile:

$$
d_{i}\left(x_{c p m l}\right)=d_{0}\left(\frac{x_{c p m l}}{L}\right)^{2},
$$

where $x_{\text {cpml }}$ ranges from 0 to the absorbing layer width $L$. The parameter $d_{0}$ is given by

$$
d_{0}=\frac{-3 C_{\max } \log R_{0}}{2 L}
$$

where $R_{0}$ is the prescribed reflection coefficient for normal incidence, which was chosen to be $10^{-7}$.

The implementation of the CPML in time domain results in a convolution between auxiliary fields, $\zeta_{i}(t)$, with the partial spatial derivatives of the electric and magnetic wavefields. The auxiliary fields obey the differential equations

$$
\frac{\partial \zeta_{i}(t)}{\partial t}-\gamma_{i} \zeta_{i}(t)=-\frac{\sigma_{i j}}{k_{i}^{2}} \frac{\partial \psi_{k}(x, t)}{\partial x_{j}}
$$




\section{Adjoint state method in fictitious domain}

The inversion of CSEM data aims to estimate a resistivity model of subsurface that explains the observed data. EM inversion is a nonlinear problem and usually is solved as an iterative process. In Quasi-Newton implementations, at each iteration the resistivity model is updated based on the gradient of the misfit function (Støren et al., 2008). In order to present the adjoint method we follow Chavent (2010). We start from the least-squares misfit functional, $\chi$,

$$
\begin{array}{r}
\chi\left(E_{i}, H_{i}, \sigma_{i j}\right)=\frac{1}{2} \sum_{s} \sum_{r} \sum_{\omega} \sum_{i=1}^{3} W^{E} \mid E_{i}^{o b s}\left(x^{r}, x^{f}, \omega\right) \\
-\left.E_{i}\left(x^{r}, x^{s}, \omega \mid \sigma_{i j}\right)\right|^{2} \\
+\frac{1}{2} \sum_{f} \sum_{r} \sum_{\omega} \sum_{i=1}^{3} W^{H} \mid H_{i}^{o b s}\left(x^{r}, x^{s}, \omega\right) \\
-\left.H_{i}\left(x^{r}, x^{s}, \omega \mid \sigma_{i j}\right)\right|^{2},
\end{array}
$$

where the first summation over $s$ is associated with all source positions, the summation over all receiver positions are represented by index $r$, the summation over all recorded frequencies are represented by $\omega$ and, the summation over all recorded field components is represented by index $i . W^{E}$ and $W^{H}$ are weighting functions which penalize the misfit between the modeled and observed EM field components (Plessix and Mulder, 2008); $E_{i}^{o b s}\left(\omega, \mathbf{x}_{r}, \mathbf{x}_{f}\right)$ corresponds to the $i$-th component of the observed electric field at the receiver position $x_{r}$, due to a source located at $x_{s}$, for frequency $\omega ; E_{i}\left(\omega, \mathbf{x}_{r}, \mathbf{x}_{f}\right)$ is the corresponding modeled component, analogously for $H_{i}^{\text {obs }}\left(\omega, \mathbf{x}_{r}, \mathbf{x}_{f}\right)$ and $H_{i}\left(\omega, \mathbf{x}_{r}, \mathbf{x}_{f}\right)$.

The adjoint state method can be understood as an extension of the Lagrange multipliers method. For each Maxwell equation in frequency domain, here formally there is an associated adjoint field, $\lambda_{i}^{E}, \lambda_{i}^{H}$, such that we can specify an Lagrangian unconstrained functional (Chavent, 2010),

$$
\begin{aligned}
& \mathscr{L}\left(E_{i}, H_{i}, \sigma_{i j}, \lambda_{i}^{E}, \lambda_{i}^{H}\right)= \\
& \frac{1}{2} \sum_{f} \sum_{r} \sum_{\omega}\left\{\sum_{i=1}^{3} W^{E}\left|E_{i}^{o b s}\left(x^{r}, x^{f}, \omega\right)-E_{i}\left(x^{r}, x^{f}, \omega \mid \sigma_{i j}\right)\right|^{2}\right. \\
& \left.+\sum_{i=1}^{3} W^{H}\left|H_{i}^{o b s}\left(x^{r}, x^{f}, \omega\right)-H_{i}\left(x^{r}, x^{f}, \omega \mid \sigma_{i j}\right)\right|^{2}\right\} \\
& +\mathscr{R} e\left\{\sum_{f} \sum_{\omega} \int_{\Omega(x)} d \Omega \lambda_{i}^{E}\left(x, x^{f}, \omega\right)\right. \\
& \left.\left[-\varepsilon_{i j k} \frac{\partial H_{k}(x, \omega)}{\partial x_{j}}+\sigma_{i j}(x) E_{j}(x, \omega)+J_{i}(x, \omega)\right]\right\} \\
& +\mathscr{R} e\left\{\sum_{f} \sum_{\omega} \int_{\Omega(x)} d \Omega \lambda_{i}^{H}\left(x, x^{f}, \omega\right)\right. \\
& \left.\left[\varepsilon_{i j k} \frac{\partial E_{k}(x, \omega)}{\partial x_{j}}-i \omega \mu H_{i}(x, \omega)+K_{i}(x, \omega)\right]\right\}
\end{aligned}
$$

in which $\mathscr{R} e$ indicates the real part of a complex number.

In order to compute the gradient of the Lagrangean functional we compute the first order perturbations relative to the EM fields and conductivity components. Requiring the Lagrangian to be independent of the first order EM field perturbations, we obtain the system of equations for the adjoint fields $\lambda^{E}$ and $\lambda^{H}$ :

$$
\begin{aligned}
& -\varepsilon_{i j k} \frac{\partial \lambda_{k}^{H}(x, \omega)}{\partial x_{j}}+\sigma_{i j} \lambda_{j}^{E}(x, \omega)= \\
& -\sum_{r} \delta\left(x-x^{r}\right) W^{E}\left[E_{i}^{o b s}\left(x^{r}, x^{f}, \omega\right)-E_{i}\left(x^{r}, x^{f}, \omega\right)\right], \\
& \varepsilon_{i j k} \frac{\partial \lambda_{k}^{E}(x, \omega)}{\partial x_{j}}-i \omega \mu \lambda_{i}^{H}(x, \omega)= \\
& -\sum_{r} \delta\left(x-x^{r}\right) W^{H}\left[H_{i}^{o b s}\left(x^{r}, x^{f}, \omega\right)-H_{i}\left(x^{r}, x^{f}, \omega\right)\right] .
\end{aligned}
$$

and the gradient of the misfit functional relative to the conductivity tensor components,

$$
\left.\frac{\partial \chi}{\partial \sigma_{i j}}\right|_{\mathbf{x}}=\mathscr{R} e\left\{\sum_{f} \sum_{\omega} \lambda_{i}^{E}(x, \omega) E_{j}(x, \omega)\right\} .
$$

Threfore, the determination of the gradient for leastsquares misfit functional requires two modeling phases. One to determine the electromagnetic fields and another to compute the corresponding adjoint fields, $\lambda^{E}$ and $\lambda^{H}$. Finally, the gradient at each point of the model domain can be obtained by equation 32 .

In order to use the correspondence principle to solve the adjoint equations (30) and (31) we need to estimate a correspond current density vector source in time domain that honors the misfit measured for the observed frequencies at every single receiver. Specifically, we need to solve the following linear system resulting from the discretization of equation (17),

where

$$
E_{k}\left(x_{r}, \omega_{m}\right)=A_{m n} E_{k}^{\prime}\left(x_{r}, t_{n}^{\prime}\right)
$$

$$
A_{m n}=\sum_{n=1}^{N_{t}} \Delta t^{\prime} e^{-\sqrt{\omega_{m} \omega_{0}} t_{n}^{\prime}} e^{i \sqrt{\omega_{m} \omega_{0}} t_{n}^{\prime}},
$$

where $m$ is the current index of the frequencies we wish to adjust, $N_{\omega}$ is the number of frequencies we wish to investigate, and $N_{t}$ is the number of intervals of the time evolution of the experiment. In matrix notation equation (33) can be written as

$$
E\left(x_{r}, \omega\right)=A E^{\prime}\left(x_{r}, t^{\prime}\right) \text {. }
$$

This problem is highly underdetermined and therefore does not have a unique solution.

In order to obtain a solution to system (33), we use a preconditionig by reparameterization (Claerbout, 2003). We chose a smoothing preconditioning operator, $P$, in wich each row correspond to a Ricker pulse,

$$
P_{n k}=\left(1-2 \pi f_{p}\left(t_{n}-t_{k}\right)^{2}\right) e^{-\pi f_{p}\left(t_{n}-t_{k}\right)^{2}},
$$

where $f_{p}$ is a user prescribed peak frequency. To assure stability we also add a damping equation such that the solution is achieved by solving problem,

$$
\left(\begin{array}{c}
A P \\
\lambda_{t} I
\end{array}\right) y=\left(\begin{array}{c}
E \\
0
\end{array}\right)
$$

by least-squares conjugate gradient, where $y$ is an auxiliar reparametrization vector. Finally, the fictitious source is obtained, $E^{\prime}=P y$.

This method produces a pulse in the fictitious time domain, which is causal and fits accurately the observed misfit spectrum. 


\section{Validation}

To validate the algorithm we compared the results obtained from a 2.5D modeling in the time domain with results of a modeling in the frequency domain, Finite Elements. We used a model with $30 \mathrm{~km}$ in the east-west direction, $20 \mathrm{~km}$ in the north-south direction and $14 \mathrm{~km}$ of depth, figure 1.

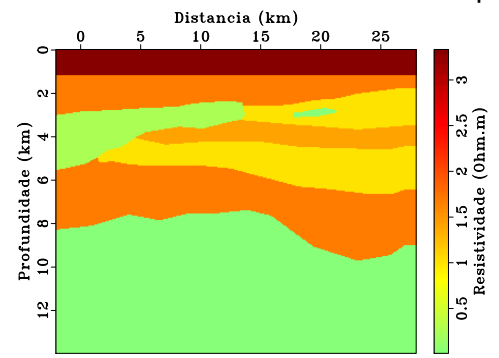

Figure 1: Resistivity model used for 2.5D validation modeling.

For modeling, we used a spatial discretization of $50 \mathrm{~m}$ in the $z$ direction, and $100 \mathrm{~m}$ in the $x$ and $y$ directions, with receivers at every $100 \mathrm{~m}$ in the $x$ direction arranged in the center of the model with respect to the $y$ axis, at a depth of $950 \mathrm{~m}$ from the surface of the model, and a source in the center of the model, at a $1000 \mathrm{~m}$ of depth. The temporal sampling of the fields was $20 \mathrm{~ms}$. The comparison between the results obtained from the two methods is shown in figure 2 .
Amplitude

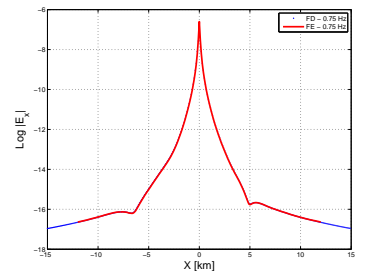

Phase

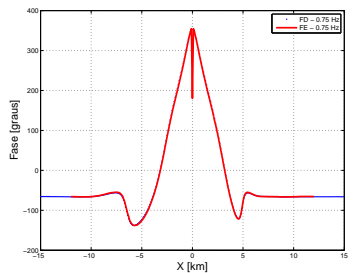

Figure 2: Time domain modeling by Finite Differences, blue curve. Modeling in the frequency domain by Finite Elements, red curve, for the frequency $0.75 \mathrm{~Hz}$.

The amplitude and phase of the fields were well behaved and had similar results to both methods, proving the effectiveness of $2.5 \mathrm{D}$ modeling in the time domain using the correspondence principle.

\section{Results}

In order to evaluate the effectiveness of the developed algorithm for modeling complex environments with anisotropic conductivity and complex geometry, we used the Marlim R3D (MR3D) model, developed by Carvalho and Menezes (2017), where high resistivity regions are associated to turbiditic sands, where there is probably an oil reservoir, immersed in a low resistivity environment, where the deeper regions of the model are marked by salt deposits.

For the computational implementation, we reduced the width of the water layer to $500 \mathrm{~m}$ and eliminated the effects of bathymetry, assigning to all points of the model above to $750 \mathrm{~m}$ the sea water resistivity. In addition, we assign a limiting resistivity of $160 \Omega . m$, since hydrocarbon reservoirs, our research target, generally have up to $100 \Omega . m$ of resistivity. Finally, we reasample the model in a grid of $100 \mathrm{~m}$ extension along the $x$ and $y$ axes, and $50 \mathrm{~m}$ extension in the $z$ axis, figure 3 . Horizontal resistivity

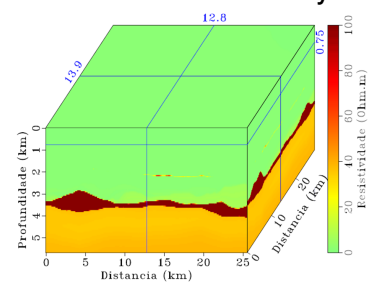
Vertical resistivity

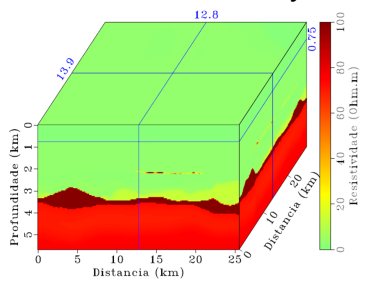

Figure 3: Horizontal and vertical resistivity models adapted from the Marlim R3D model.

We used an acquisition geometry with 25 (twenty five) sources located at the real receivers depth, $750 \mathrm{~m}$, arranged in the center of the model related to the $x$ and $y$ axes, equally spaced each other of $1020 \mathrm{~m}$, with a minimum offset between the source and origin of the model of $510 \mathrm{~m}$, emitting a pulse for $15.7 \mathrm{~s}$. The receivers were arranged in the real sources depth, $700 \mathrm{~m}$, in the center of the model with respect to the $y$ axis, at each point of the grid. Figure 4 illustrates the amplitude and phase of the electric fields for the frequencies $0.1,0.75$ and $1.25 \mathrm{~Hz}$.

Amplitude - x component

Phase - $\mathrm{x}$ component
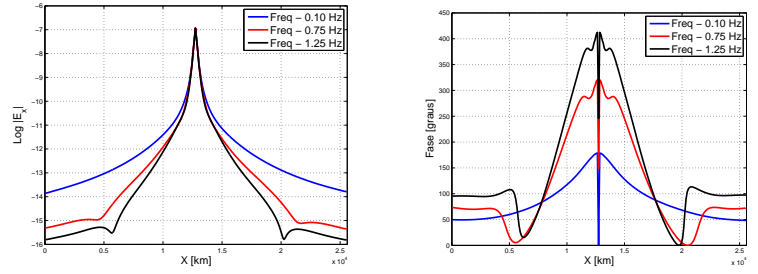

Amplitude - y component

Phase - y component
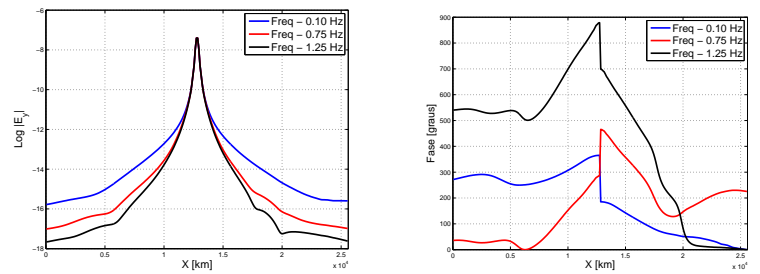

Figure 4: Amplitude and phase of the electric field, due to the model based on the Marlim R3D model, for the frequencies $0.1,0.75$ and $1.25 \mathrm{~Hz}$.

As we can see, the fields are well behaved. In smallest frequencies, the amplitude and the skin depth of the fields are greater. In the phase component it is possible to identify the critical distance from which the air-waves dominate the signal in front of the subsurface structures, which decreases with the increasing of frequency. For the modeling of the computed data, we apply a smoothing to the model, and from the residue between the two data we obtain the source of the adjoint fields.

To obtain the adjoint source we solve the linear system in equation 38. In order to apreciate the differences between our proposal and the method used by Mittet (2010), figure 5 presents the corresponding fitted spectrum using both methodologies. The pulse obtained by the conjugated gradient methods, preconditioned by Ricker pulse, blue line, produced better fitting at all the observed data in frequency domain. 


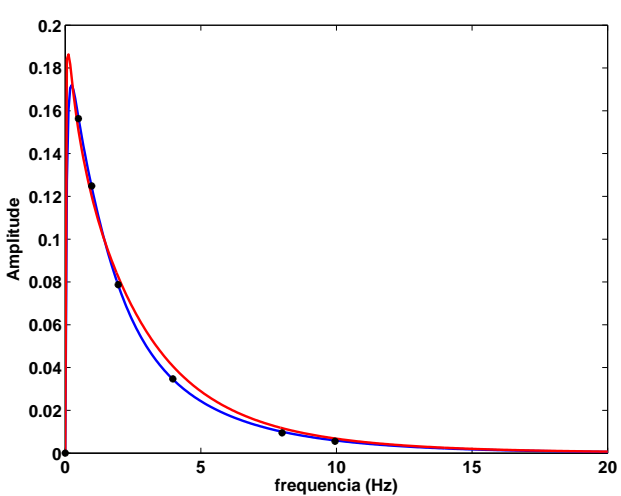

Figure 5: Source pulse on frequency domain, where the black points are the observed frequencies.

Figure 6 illustrates the gradient due to the vertical component of the conductivity tensor, resulting from the cross-correlation between the computed and adjoint electric fields. Gradient $-0.10 \mathrm{~Hz} \quad$ Gradient $-0.75 \mathrm{~Hz}$
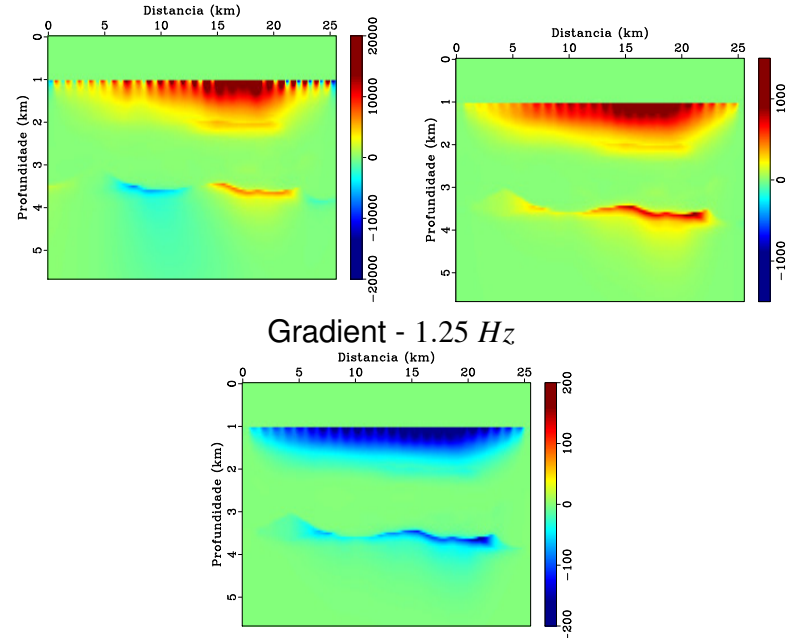

Figure 6: Gradient for the $z$ component of the conductivity tensor for the model based on Marlim R3D.

The gradient due to the horizontal components of the conductivity tensor is less sensitive to variations in the physical property of the model than the gradient due to the vertical component of the conductivity tensor. We also note that as the frequency increases, the amplitude of the scale decreases. In addition, we can see that both the salt layer and the turbiditic reservoir appear to have been better imaged at the frequency $0.75 \mathrm{~Hz}$, which probably is due to the intermediate depth of these targets.

\section{Conclusion}

We presented the implementation of forward and adjoint EM modeling using the Correspondence Principle. Our main contributions for the algorithm are in the implementation of effective CPML boundary conditions and an new methodology to estimate the source pulse in fictitious domain given a finite number of samples of the EM spectrum at each receiver. The estimated pulse has, in general, smaller width that the pulses estimated using the semi-analytical method proposed by Mittet (2010). Moreover, in our numerical experiments, we also obtained a better fitting of the receivers data in frequency domain.
We derived the computation of the gradient for the objective function least-squares CSEM inversion using forward and adjoint modeling in the fictitious domain. After the validation of the algorithm in simple $2.5 \mathrm{D}$ and $3 \mathrm{D}$ models, we applied the methodology in the Marlim R3D model. We evaluated the gradient misfit functional around a smoothed version of the exact model. The images of the gradient for each component of the conductivity tensor show that the resolution of the vertical component of the conductivity tensor is higher that the resolution of horizontal components. This result is in accordance with Støren et al. (2008). Finally, the gradient was able to resolve resistivity variations for a large depth range. Moreover, the gradient of conductivity vertical was able identify the reservoir region.

\section{Acknowledgments}

To the professors Cícero Regis and Maria Amélia Novais Schleicher for the discussions, to the geophysicist Deivid Nascimento for contributions to the algorithm, PETROBRAS and CNPq for the financial support and the CPGf / UFPA for the support to the research.

\section{References}

Carvalho, B. R., and P. T. L. Menezes, 2017, Marlim r3d: a realistic model for csem simulations-phase i: model building: Brazilian Journal of Geology, 47, 633-644.

Chavent, G., 2010, Nonlinear least squares for inverse problems - theoretical foundations and step-by-step guide for applications: Springer Netherlands. Scientific Computation, No. 1.

Claerbout, Jon F. e Fomel, S., 2003, Image estimation by example: Geophysical soundings image construction multidimensional autoregression.

de Hoop, A. T., 1996, A general correspondence principle for time-domain electromagnetic wave and diffusion fields: Geophysical Journal International, 127, 757-761.

de la Kethulle de Ryhove, S., and R. Mittet, 2014, 3d marine magnetotelluric modeling and inversion with the finite-difference time-domain method: Geophysics, 79, E269-E286.

Holberg, O., 1987, Computational aspects of the choice of operator and sampling interval for numerical differentiation in large-scale simulation of wave phenomena: Geophysical prospecting, 35, 629-655.

Komatitsch, D., and R. Martin, 2007, An unsplit convolutional perfectly matched layer improved at grazing incidence for the seismic wave equation: Geophysics, 72, SM155-SM167.

Lee, K. H., G. Liu, and H. Morrison, 1989, A new approach to modeling the electromagnetic response of conductive media: Geophysics, 54, 1180-1192.

Maaø, F. A., 2007, Fast finite-difference time-domain modeling for marine-subsurface electromagnetic problems: Geophysics, 72, A19-A23.

Mittet, R., 2010, High-order finite-difference simulations of marine csem surveys using a correspondence principle for wave and diffusion fields fdtd simulation of marine csem surveys: Geophysics, 75, F33-F50.

Plessix, R.-E., and W. Mulder, 2008, Resistivity imaging with controlled-source electromagnetic data: Depth and data weighting: Inverse problems, 24, 034012.

Roden, J. A., S. D. Gedney, et al., 2000, Convolutional pml (cpml): An efficient fdtd implementation of the cfs-pml for arbitrary media: Microwave and optical technology 
letters, 27, 334-338.

Schild, S., 2009, Advanced material modeling in em-fdtd: PhD thesis, ETH Zurich.

Støren, T., J. Zach, and F. Maaø, 2008, Gradient calculations for $3 d$ inversion of csem data using a fast finite-difference time-domain modelling code: Presented at the 70th EAGE Conference and Exhibition incorporating SPE EUROPEC 2008.

Wang, T., and G. W. Hohmann, 1993, A finitedifference, time-domain solution for three-dimensional electromagnetic modeling: Geophysics, 58, 797-809.

Yee, K., 1966, Numerical solution of initial boundary value problems involving maxwell's equations in isotropic media: IEEE Transactions on antennas and propagation, 14, 302-307. 